\title{
FUNCTION THEORETIC RESULTS FOR COMPLEX INTERPOLATION FAMILIES OF BANACH SPACES
}

BY

RICHARD ROCHBERG ${ }^{1}$

\begin{abstract}
The theory of complex interpolation of Banach spaces is viewed as a branch of the theory of vector valued holomorphic functions. Versions of the Schwarz lemma, Liouville's theorem, the identity theorem and the reflection principle are proved and are interpreted from the point of view of interpolation theory.
\end{abstract}

I. Introduction and summary. The fundamental construction in the theory of complex interpolation of finite-dimensional Banach spaces produces families of Banach spaces $\left\{B_{z}\right\}$ parametrized by the points $z$ of a domain $D$ in the complex plane. One feature of the spaces constructed is that a vector-valued analytic function $f$ taking values in the $\left\{B_{z}\right\}$ (i.e. $f(z)$ in $B_{z}$ ) will satisfy a maximum principle. The fundamental estimates in the theory of complex interpolation of Banach spaces follow from this function theoretic maximum principle. In this paper we study other aspects of function theory for this type of vector-valued analytic function. We obtain analogs of the Schwarz lemma, of Liouville's theorem, of the identity theorem and of the reflection principle. In general, once the appropriate analogy is established the proofs follow their classical counterparts. In several cases the results obtained are closely related to known results in interpolation theory.

In $§ I I$ we review the ideas of and establish the notation for our discussion of complex interpolation of Banach spaces.

In $\S$ III we present a "Schwarz lemma". That is, we give a local estimate for the modulus of continuity of the norm function of $B_{z}$ as a function of $z$. The estimate we obtain is similar to estimates used by Zafran and Sneiberg in studying the spectrum of operators defined on interpolation families of Banach spaces. We use the lemma to bound the modulus of continuity of the map which associates to the point $z$ in $D$ the Banach space $B_{z}$. This map is regarded as a map from the domain $D$ with its hyperbolic geometry into the set of all Banach spaces (of a given dimension) regarded as a metric space with the Banach-Mazur metric.

Two variations on Liouville's theorem are given in $\S \mathrm{IV}$. In both it is assumed that $\left\{B_{z}\right\}$ is a complex interpolation family defined on the entire complex plane. In the first case the conclusion is that all the $B_{z}$ are isometrically isomorphic. The second conclusion, requiring further hypotheses. is that this isometric isomorphism is implemented by the identity map.

In $\S \mathrm{V}$ we present an identity theorem for interpolation families. The result is more in the spirit of the identity theorem for harmonic functions than that for

Received by the editors February 8, $198: 3$ and, in revised form. July 18. 198:3.

1980 Mathematics Subject ('lossification. Primary 30( $80.46 \mathrm{E} 40.46 \mathrm{M} 35$ ).

${ }^{1}$ This work was supported in part by N.SF (irant M( $\mathrm{S}-8002689$ ).

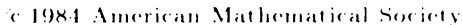
$0002-9917 \times 1 \$ 100+\$ 25$ por pase. 
holomorphic functions - two interpolation families which agree on an open subset of their common domain of definition agree throughout the domain. This result gives a new approach to the recent four-space reiteration theorem of $\mathrm{T}$. Wolff.

In $\S \mathrm{VI}$ we present two variations of the reflection principle for interpolation families. The first version which applies to $l^{p}$ spaces (and other one-parameter families) is based on the reflection principle for harmonic functions. The second version involves reflection of an interpolation family with respect to a fixed Hilbert space which forms the boundary values of the family on an interval in the boundary. Roughly the reflected family is the family of dual spaces of the given Banach spaces with respect to the duality pairing determined by the boundary Hilbert space. This extends work by S. G. Krein and Y. I. Petunin on construction of scales of Banach spaces containing three given spaces. Some discussion is given to the problem of reflecting across boundary values which are not Hilbert spaces.

We should emphasize that all of the analysis we give is for finite-dimensional Banach spaces. There are two reasons for this beyond technical convenience. First, much of infinite-dimensional interpolation theory deals with Banach spaces defined up to equivalent norms (i.e. an isomorphic rather than isometric theory). Most of the results here deal with the isometric theory and do not have clear analogs in the isomorphic theory. Second, many of the arguments in this paper use the existence of certain "extremal functions". Such functions need not exist in the infinite-dimensional theory (although they do in many important cases). The validity of these results in the infinite-dimensional case has not been studied.

Our discussion is in the context of interpolation theory. The operator theoretic significance of these results is not clear. One result of this type which does have direct operator theoretic significance is the Cauchy estimates for the derivative of an analytic function. That estimate is developed and studied systematically in [9].

II. Interpolation. The vector spaces we consider will all be finite dimensional. By a Banach space we will mean $\mathbf{C}^{n}$ equipped with a norm function $\|\cdot\|$. For a family of Banach spaces $\left\{B_{z}\right\}$ indexed by $z$, we will use the same subscripts for the norm. Thus the norm of $B_{z}$ will be denoted $\|\cdot\|_{z}$ or $\|\cdot\|_{B_{z}}$. For $1 \leq p<\infty$ we denote by $l_{n}^{p}$ the space $\mathbf{C}^{n}$ with the norm $\left\|\left(v_{1}, \ldots, v_{n}\right)\right\|_{p}=\left(\sum\left|v_{i}\right|^{p}\right)^{1 / p} . l_{n}^{\infty}$ will denote $\mathbf{C}^{n}$ with the norm $\left\|\left(v_{1}, \ldots, v_{n}\right)\right\|_{\infty}=\max \left|v_{i}\right|$. If there is a complex linear norm-preserving map of the Banach space $B$ to $l_{n}^{2}$ we will call $B$ a Hilbert space. We denote the dual of a Banach space $B$ by $B^{*}$ and establish the duality with respect to the bilinear (not the sesquilinear) pairing. Thus

$$
\left\|\left(v_{1}, \ldots, v_{n}\right)\right\|_{B^{*}}=\sup \left\{\left|\sum v_{i} w_{i}\right| ;\left\|\left(w_{1}, \ldots, w_{n}\right)\right\|_{B}=1\right\} .
$$

We now present without proof the main features of the theory of complex interpolation of finite-dimensional Banach spaces. More details, including proofs of the statements in this section and applications of this theory, are in $[\mathbf{3}, \mathbf{4}$ and $\mathbf{5}]$. A comprehensive view of interpolation theory can be found in $[\mathbf{1}$ or $\mathbf{2}]$.

Let $D$ be an open subset of $\mathbf{C}$. Let $\operatorname{Hol}(D)$ be the space of holomorphic $\mathbf{C}^{n}$ valued functions defined on $D$. We regard $D \times \mathbf{C}^{n}$ as a family of vector spaces parametrized by points of $D$. Suppose that for each $z$ in $D$ there is specified a norm function $\|\cdot\|_{z}$ defined on the vector space $\{z\} \times \mathbf{C}^{n}=\mathbf{C}^{n}$. Generally this norm will vary from point to point. We denote the normed vector space $\left(\mathbf{C}^{n},\|\cdot\|_{z}\right)$ by $B_{z}$. Thus $\left\{B_{z}\right\}_{z \in D}$ is a family of Banach spaces parametrized by points of $D$. 
We say the family $\left\{B_{z}\right\}$ is continuous if the function $\|v\|_{z}$ is continuous for $(z, v)$ in $D \times \mathbf{C}^{n}$. We say that $\left\{B_{z}\right\}$ is a subinterpolation family on $D$ if given any $F$ in $\operatorname{Hol}(D), \log \|F(z)\|_{z}$ is a subharmonic function of $z$ for $z$ in $D$. If the family of dual spaces $\left\{B_{z}^{*}\right\}$ also form a subinterpolation family then we say that $\left\{B_{z}\right\}$ is a (complex) interpolation family.

The results of $[\mathbf{4}]$ insure the existence of interpolation families with given boundary values. Start with a smooth simple closed curve $\Gamma$ which bounds a region $D$ and with a norm function $\|\cdot\|_{z}$ specified for each $z$ in $\Gamma$. We suppose that $\|\cdot\|_{z}$ is a continuous function of $z$ and that there are constants $C_{1}, C_{2}$ so that $C_{1} \leq\|v\|_{z} /\|v\|_{2} \leq C_{2}$ for all $v$ in $\mathbf{C}^{n}$, for all $z$ in $\Gamma$ (these assumptions are unnecessarily strict). Then there is a unique interpolation family $\left\{B_{z}\right\}$ defined on $D$ which has the given $\|\cdot\|_{z}$ as its boundary values. (Here boundary values are taken in the sense of nontangential convergence of the norm function on a set of $\Gamma$ of full measure with respect to arc length.) Furthermore if $\left\{D_{z}\right\}$ is any subinterpolation family on $D$ which extends to $\Gamma \cup D$ and satisfies for all $z$ in $\Gamma$

$$
\|v\|_{D_{z}} \leq\|v\|_{z} \quad \forall v \in \mathbf{C}^{n}
$$

then (2.1) also holds for all $z$ in $D$. (This is a version of the fundamental maximum principle mentioned in the introduction.) Finally, given $z_{0}$ in $D$ and $v$ in $\mathbf{C}^{n}$ there is an extremal function for $v$ at $z_{0}$; that is, there is an $F$ in $\operatorname{Hol}(D)$ which satisfies $F\left(z_{0}\right)=v$ and $\|F(z)\|_{z}=\|v\|_{z_{0}}$ for all $z$ in $D$. The fundamental step in the construction of the norm on $B_{z}$ is to define $\|\cdot\|_{z_{0}}$ for $z_{0}$ in $D$ by setting, for $v$ in $\mathbf{C}^{n}$,

$$
\|v\|_{z_{0}}=\inf \left\{\sup _{z \in \Gamma}\|F(z)\|_{z} ; F \in \operatorname{Hol}(\bar{D}), F\left(z_{0}\right)=v\right\} .
$$

The extremal functions are those for which the infimum in (2.2) is attained.

In one dimension the situation is simple. The norm function $\|\cdot\|_{z}$ is fully specified by the positive function $w(z)=\|1\|_{z}$. A family of Banach spaces will be a subinterpolation family exactly if $\log w(z)$ is subharmonic. Since $\|1\|_{z}^{*}=w(z)^{-1}$, the family is an interpolation family exactly if $\log w(z)$ is harmonic. In this case the existence theorem of the previous paragraph becomes an existence theorem for the Dirichlet problem for the equation $\Delta \log w=0$.

In $n$ dimensions, one class of examples is especially easy to describe. Suppose $\|\cdot\|$ is given and that $T_{z}$ is a family of invertible linear maps of $\mathbf{C}^{n}$ to $\mathbf{C}^{n}$ which vary analytically with $z$ for $z$ in $D$. Define $\|\cdot\|_{z}$ by $\|v\|_{z}=\left\|T_{z} v\right\|$. The family $\left\{B_{z}\right\}$ defined by $B_{z}=\left(\mathbf{C}^{n},\|\cdot\|_{z}\right)$ is an interpolation family. We call families of this form flat (some geometric consequences of this hypothesis are in [8]). Every one-dimensional interpolation family is locally flat. That is because every solution of $\Delta \log w(z)=0$ is locally of the form $w(z)=|f(z)|$ for some holomorphic function $f$. The same is true in higher dimension for Hilbert spaces. If the norms specified on a curve are all Hilbert space norms then the interpolation family with those boundary values will consist entirely of Hilbert spaces and will form a flat family. (This is proved in $[\mathbf{4}]$. The crucial fact is that the extremal function for $v$ at a point $z_{0}$ depends linearly on $v$.)

Not every interpolation family is flat. For an open set $D$ and $p(z)$ a function on $D$ which satisfies $1 \leq p(z) \leq \infty$, the family $\left\{B_{z}\right\}_{z \in D}$ given by $B_{z}=l_{n}^{p(z)}$ will be 
an interpolation family exactly if $1 / p(z)$ is harmonic. Because $l_{n}^{p_{1}}$ and $l_{n}^{p_{2}}$ are not linear isometric for $p_{1} \neq p_{2}$ this cannot be a flat family.

III. A Schwarz lemma. Let $D$ be the open unit disk, $\Gamma$ the unit circle, and $\left\{B_{z}\right\}$ an interpolation family on $D$. Informally, it seems reasonable that for fixed $v$ in $\mathbf{C}^{n},\|v\|_{z}$ should be a smooth function of $z$ for $z$ away from $\Gamma$. Simple examples with $n=1$ show that this smoothness is not absolute but must depend on the family $\left\{B_{z}\right\}$. The following variation on Schwarz's lemma is one way of making this precise.

THEOREM 3.1. Suppose $\left\{B_{z}\right\}$ is an interpolation family in an open set containing $D \cup \Gamma$. There is a constant $c$ so that for all $v$ in $\mathbf{C}^{n}$ and all $z$ in $D$ with $|z|<1 / 2$

$$
\left|\|v\|_{0}-\|v\|_{z}\right| \leq c|z| \int_{\Gamma}\|v\|_{e^{i \theta}} d \theta .
$$

Proof. Pick and fix $v$ in $\mathbf{C}^{n}$. Let $v(z)$ be a function in $\operatorname{Hol}(\bar{D})$ which is extremal for $v$ at 0 . Thus $v(0)=v$ and

$$
\begin{gathered}
\|v(z)\|_{z}=\|v\|_{0}, \quad z \in \bar{D} \\
\left|\|v\|_{0}-\|v\|_{z}\right|=\left|\|v(0)\|_{0}-\|v(0)\|_{z}\right|=\left|\|v(z)\|_{z}-\|v(0)\|_{z}\right| .
\end{gathered}
$$

The last equality by (3.2). Let $A$ denote the left-hand side of (3.1). By the triangle inequality we can continue the previous estimate with $A \leq\|v(0)-v(z)\|_{z}$. Now $H(z)=(v(0)-v(z)) / z$ is in $\operatorname{Hol}(\bar{D})$ and hence $\log \|H(z)\|_{z}$ and thus also $\|H(z)\|_{z}$ are subharmonic. Thus

$$
\begin{aligned}
A & \leq\|z H(z)\|_{z}=|z|\|H(z)\|_{z} \\
& \leq|z| \int_{\Gamma}\left\|H\left(e^{i \theta}\right)\right\|_{e^{i \theta}} P_{z}(\theta) d \theta / 2 \pi,
\end{aligned}
$$

where $P_{z}(\theta)$ is the Poisson kernel for evaluating at $z$. For $z$ restricted to $|z| \leq 1 / 2$ the $P_{z}(\theta)$ are uniformly bounded by some constant $c$ and hence

$$
\begin{aligned}
A & \leq c|z| \int_{\Gamma}\left\|v(0)-v\left(e^{i \theta}\right)\right\|_{e^{i \theta}} d \theta / 2 \pi \\
& \leq c|z| \int_{\Gamma}\left(\|v\|_{e^{i \theta}}+\left\|v\left(e^{i \theta}\right)\right\|_{e^{i \theta}}\right) d \theta / 2 \pi .
\end{aligned}
$$

By (3.2), $\left\|v\left(e^{i \theta}\right)\right\|_{e^{i \theta}}=\|v\|_{0}$ and by the mean value inequality for the subharmonic function $\|v\|_{z}$ this quantity is dominated by $\int_{\Gamma}\|v\|_{e^{i \theta}} d \theta / 2 \pi$. Thus, if we increase the constant in the previous estimate we may drop the second term in the integration. This proves the theorem.

Variations on this result, such as a conformally invariant version and a limiting version as $z$ tends to zero, can also be obtained. It is not clear what the best possible estimates would be in this context. The estimate (3.1) is not sharp in its form. A sharper (but more awkward) result can be obtained using the fact that $\log \|H(z)\|_{z}$ (rather than $\|H(z)\|_{z}$ ) is subharmonic.

Estimates such as (3.1) can be used as the starting point for studying how various properties of the $B_{z}$ vary with $z$. For example, in the work of Zafran and of Sneiberg (see $[\mathbf{1 1}]$ and the references there) on how the spectrum of an operator defined on 
a family of spaces varies with the space, a fundamental analytical tool (Lemma 2.4 of $[\mathbf{1 1}])$ is an estimate very similar to (3.1).

We will use (3.1) to estimate the Banach-Mazur distance between interpolation spaces. For Banach spaces $B_{1}$ and $B_{2}$ define the Banach-Mazur distance between $B_{1}$ and $B_{2}, d\left(B_{1}, B_{2}\right)$, by

$$
d\left(B_{1}, B_{2}\right)=\log \inf \left\{\|T\|\left\|T^{-1}\right\| ; T \text { an invertible linear map of } B_{1} \text { to } B_{2}\right\} .
$$

We let $B_{n}$ be the set of all $n$ dimensional Banach spaces modulo the equivalence relation of being isometrically equivalent by a linear map. $\left(B_{n}, d(\cdot, \cdot)\right)$ is a complete metric space. For more about $d(\cdot, \cdot)$ see $[\mathbf{7}]$ and the references there.

THEOREM 3.2. Let $\left\{B_{z}\right\}$ be an interpolation family on an open set containing $D \cup \Gamma$. There is a constant $c_{n}$ which depends only on $n$ so that for all $z$ in $D$

$$
d\left(B_{0}, B_{z}\right) \leq c_{n}|z| .
$$

PROOF. If all the $B_{z}$ are Hilbert spaces then $d\left(B_{0}, B_{z}\right) \equiv 0$ and the result is trivial. We will show that the general case can be approximated by this case. The crucial geometric step is the following result of F. John.

LEMMA. The metric space $\left(B_{n}, d\right)$ has finite diameter (which depends on $n$ ).

PROOF. See $\S 16$ of $[7]$.

By the Lemma, it suffices to prove (3.3) for sufficiently small $z$. Again by the Lemma, there is constant a $c$ (which depends only on $n$ ) and a family of Hilbert space norms $N_{z}(\cdot), z$ in $\Gamma$, which approximate $\|\cdot\|_{z}$ on $\Gamma$ in the sense that for all $z$ in $\Gamma$ and all $v$ in $\mathbf{C}^{n}, v \neq 0$

$$
1 / c \leq N_{z}(\cdot) /\|v\|_{z} \leq c .
$$

We now modify the family $N_{z}$ to produce a new family of norms which vary continuously with $z$. Using the continuity of $\|v\|_{z}$ as a function of $z$, we have that for each $z$ there is an open neighborhood $U(z)$ of $z$ in $\Gamma$ such that for all $v$ in $\mathbf{C}^{n}$, $v \neq 0$, and for all $w$ in $U(z)$

$$
1 / 2 c \leq N_{z}(\cdot) /\|v\|_{w} \leq 2 c .
$$

Let $U\left(z_{1}\right), \ldots, U\left(z_{k}\right)$ be a finite cover of $\Gamma$ and let $\varphi_{1}, \ldots, \varphi_{k}$ be a smooth partition of unity subordinate to that cover. For $z$ in $\Gamma$ and $v$ in $\mathbf{C}^{n}$, let \|\|$v\|\|_{z}=$ $\left(\sum \varphi_{i}(z) N_{z_{i}}(v)^{2}\right)^{1 / 2} \cdot \||| \cdot||$ is a family of Hilbert space norms which vary smoothly with $z$ and which satisfy, for a new constant $c$, all $z$ in $\Gamma$ and all $v$ in $\mathbf{C}^{n}, v \neq 0$,

$$
1 / c \leq\|v\|\left\|_{z} /\right\| v \|_{z} \leq c .
$$

Let $\left\{C_{z}\right\}$ be the interpolation family in $D$ which has boundary norms $\|\cdot\| \|_{z}$ for $z$ in $\Gamma$. The $\left\{C_{z}\right\}$ form a flat family; that is, there is an analytic family of linear maps $\mathbf{C}^{n}$ to $\mathbf{C}^{n}$ such that $T_{0}=I$ and $\left\|\mid T_{z} v\right\|_{z}=\|\| v \|_{0}$ for all $v$ in $\mathbf{C}^{n}$ and all $z$ in $D$. Let $\left\{D_{z}\right\}$ be the family of Banach spaces obtained by putting on $\mathbf{C}^{n}$ the norms

$$
\|v\|_{D_{z}}=\left\|T_{z} v\right\|_{z}
$$

$\left\{D_{z}\right\}$ is the image of an interpolation family under an analytic family of invertible linear maps. Hence $\left\{D_{z}\right\}$ is an interpolation family. Also, $D_{0}=B_{0}$. Finally, although $B_{z}$ and $D_{z}$ are not the same normed vector space they are the same 
element in $B_{n}$. Hence to prove (3.3) it suffices to estimate $d\left(D_{0}, D_{z}\right)$. We do this by using Theorem 3.1 and the identity map on $\mathbf{C}^{n}$ as a map of $D_{0}$ to $D_{z}$. For $z$ in $\Gamma$ and $v$ in $\mathbf{C}^{n},(3.5)$ gives $\|v\|_{D_{z}} /\|v\|_{D_{0}}=\left\|T_{z} v\right\|_{0} /\|v\|_{0}$. By (2.1), the estimate (3.4) for $z$ in $\Gamma$ propagates to $D$, i.e.

$$
1 / c \leq\|\| v\left\|_{z} /\right\| v \|_{z} \leq c
$$

for all $v$ in $\mathbf{C}^{n}$ and all $z$ in $D$. (Here we use the fact that $\left\{C_{z}\right\}$ and $\left\{B_{z}\right\}$ are both interpolation families.) Combining these last two estimates we obtain

$$
\frac{1}{c^{2}} \frac{\left\|\left|T_{z} v \|\right|_{z}\right.}{\|v\| \|_{0}} \leq \frac{\|v\|_{D_{z}}}{\|v\|_{D_{0}}} \leq c^{2} \frac{\|\| T_{z} v \|_{z}}{\|v\|_{0}} .
$$

The characteristic property of $T_{z}$ is that $\left\|\mid T_{z} v\right\|_{z}=\|\| v\|\|_{0}$. Thus

$$
c^{-2} \leq\|v\|_{D_{z}} /\|v\|_{D_{0}} \leq c^{2}
$$

for all $v$ in $\mathbf{C}^{n}$ and all $z$ in $\bar{D}$. We now apply Theorem 3.1 and conclude that for small $z$

$$
\left|\|v\|_{D_{0}}-\|v\|_{D_{z}}\right| \leq c|z|\|v\|_{D_{0}}
$$

for a new constant $c$ which depends only on $n$. Thus,

$$
1-c|z| \leq\|v\|_{D_{z}} /\|v\|_{D_{0}} \leq 1+c|z| \text {. }
$$

Hence the identity map of $D_{0}$ to $D_{z}$ satisifes the operator norm estimate

$$
\log \|T\|\left\|T^{-1}\right\| \leq c|z|
$$

for a constant $c$ which depends only on the dimension. This finishes the proof.

The following invariant formulation is a direct consequence.

COROLlary 3.3. Let $\left\{B_{z}\right\}$ be an interpolation family on $D$. The map of $D$ to $B_{n}$ which sends $z$ to $B_{z}$ is a Lipschitz map from $D$ with its hyperbolic metric into $B_{n}$ with the metric d. The Lipschitz constant can be chosen to depend only on the dimension $n$.

There are many open questions related to the previous theorem. We mention two specific but typical ones. $l_{n}^{2}$ is a Hilbert space and $l_{n}^{3}$ is not. Thus $d\left(l_{n}^{2}, l_{n}^{3}\right)>0$. The previous theorem insures that if $\left\{B_{z}\right\}$ is an interpolation family on $D$ with $B_{0}=l_{n}^{2}$ and $B_{z_{0}}=l_{n}^{3}$ then there is a lower bound on $\left|z_{0}\right|$. The question is: how small can $\left|z_{0}\right|$ be? If all of the $B_{z}$ are of the form $l_{n}^{p(z)}$ then $1 / p(z)$ must be harmonic and the problem becomes a relatively elementary extremal problem for harmonic functions. The boundary values of the extremal configuration are $l_{n}^{p(z)}=l_{n}^{\infty}$ for $z$ on $\Gamma \cap\{\operatorname{Re} z>0\}$ and $l_{n}^{p(z)}=l_{n}^{1}$ for $z$ on $\Gamma \cap\{\operatorname{Re} z<0\}$. We conjecture that this is the extremal configuration for all possible interpolation families (not just those of the form $\left.B_{z}=l_{n}^{p(z)}\right)$.

The second problem is to find the approprite analog of the theorem for infinitedimensional spaces. Theorem 3.2 fails. The infinite-dimensional family $l^{p(z)}$ with $1 / p(z)$ harmonic is an interpolation family and for the choice of $p(z)$ of the previous paragraph will give $B_{0}=l^{2}, B_{z_{0}}=l^{3}$. However $d\left(l^{2}, l^{3}\right)$ is infinite. A particular question would be to give a lower bound for $\left|z_{0}\right|$ among all interpolation families with $B_{0}=l^{2}$ and $B_{z_{0}}=l^{3}$. Although we suspect that this question has the same answer as the previous one, we do not know how to insure in this case that the lower bound must be positive. 
IV. Liouville's theorem. Liouville's theorem is the statement that a bounded analytic function defined on the entire complex plane is constant. Many proofs can be given for the theorem. In this section we show the results that are obtained when two of those proofs are applied to complex interpolation families of Banach spaces.

THEOREM 4.1. Suppose $\left\{B_{z}\right\}$ is an interpolation family defined on all of $\mathbf{C}$, then $\left\{B_{z}\right\}$ is flat.

Proof. For $k=1,2, \ldots$ let $D_{k}=\{z ;|z|<k\}$. For given $z_{0}$ in $\mathbf{C}$ and $v$ in $\mathbf{C}^{n}$ and for large $k$ there is a sequence of functions $F_{k}$ in $\operatorname{Hol}\left(D_{k}\right)$ which are extremal functions for $v$ at $z_{0}$ for the family $\left\{B_{z}\right\}$ on $D_{k}$ (i.e. $F_{k}\left(z_{0}\right)=v$ and $\|F(z)\|_{z}=\|v\|_{z_{0}}$ for $z$ in $D_{k}$ ). By a standard normal family argument there is a subsequence of $\left\{F_{k}\right\}$ which converges to a limit $F=F_{v}$ in $\operatorname{Hol}(\mathbf{C})$ which satisfies

$$
F_{v}\left(z_{0}\right)=v
$$

and

$$
\left\|F_{v}(z)\right\|_{z}=\|v\|_{z_{0}} .
$$

Select such an $F_{v}$ for each $v$ in $\mathbf{C}^{n}$. For $v_{1}, v_{2}$ in $\mathbf{C}^{n}$ and $\alpha_{1}, \alpha_{2}$ in $\mathbf{C}$ consider

$$
H(z)=\left(\alpha_{1} F_{v_{1}}+\alpha_{z} F_{v_{2}}-F_{\alpha_{1} v_{1}+\alpha_{2} v_{2}}\right)(z) .
$$

$H$ is in $\operatorname{Hol}(\mathbf{C})$. Using the triangle inequality and (4.2) we see that

$$
\|H(z)\|_{z} \leq 2\left(\left|\alpha_{1}\right|\left\|v_{1}\right\|_{z_{0}}+\left|\alpha_{2}\right|\left\|v_{2}\right\|_{z_{0}}\right) .
$$

Thus $\|H(z)\|_{z}$ is a bounded subharmonic function and hence is constant. Evaluating at $z_{0}$ and using (4.1) we conclude that $H(z) \equiv 0$. Thus $T_{z}(v)=F_{v}(z)$ is a linear map of $\mathbf{C}^{n}$ which is analytic as a function of $z$ and satisfies $\|v\|_{z}=\left\|T_{z}^{-1} v\right\|_{0}$ for all $z$ in $\mathbf{C}$ and all $v$ in $\mathbf{C}^{n}$. This is the required conclusion.

This theorem looks very different from the classical Liouville theorem. However if we weaken the conclusion slightly then the theorem states that the map which sends $z$ to $B_{z}$, regarded as a map from a domain in $\mathbf{C}$ into $B_{n}$, if entire, must be constant. A hypothesis of boundedness is implicit because the target set, $B_{n}$, is a bounded set (by the Lemma in the previous section).

Liouville's theorem can be regarded as a globalized version of the Schwarz lemma and can be proved using that lemma. Here is that proof.

THEOREM 4.2. Suppose $\left\{B_{z}\right\}$ is an interpolation family defined on all of $\mathbf{C}$ and that for each $v$ in $\mathbf{C}^{n},\|v\|_{z}$ is bounded. Then $\|v\|_{z}$ is constant.

PROOF. Let $z_{0}$ be picked and fixed and for fixed positive $\varepsilon$ let $\left\{C_{z}\right\}$ be the interpolation family defined by $\|v\|_{C_{z}}=\|v\|_{z / \varepsilon}$. We apply Theorem 3.1 to the family $C_{z}$ on the unit disk. Evaluating (3.1) for $z=\varepsilon z_{0}$ yields

$$
\left|\|v\|_{0}-\|v\|_{z_{0}}\right| \leq c\left|z_{0}\right| \varepsilon \int_{\Gamma}\|v\|_{e^{i \theta} / \varepsilon} d \theta / 2 \pi
$$

By hypothesis the integral on the right is bounded by a bound independent of $\varepsilon$. Thus we may take the limit as $\varepsilon$ goes to zero. This gives the desired conclusion.

In contrast to these two results, the classical proofs of Liouville's theorem using the Cauchy estimate for the derivatives (either the first derivative at all points or all derivatives at one point) do not seem to generalize directly to this context. 
V. The identity theorem. Suppose $\left\{B_{z}\right\}$ and $\left\{C_{z}\right\}$ are two interpolation families with a common domain of definition $D$. An identity theorem is a result which says that if

$$
B_{z}=C_{z}
$$

for all $z$ in a small subset of $D$ then (5.1) holds for all $z$ in $D$. In the case when the spaces are one-dimensional, (5.1) is equivalent to a statement of equality at $z$ of two harmonic functions. Thus it should not be expected that equality on, say, a segment in $D$ will insure equality throughout $D$. Here is an observation about harmonic functions which suggests a correct general result. If two harmonic functions on the unit disk agree on the circle centered at the origin with radius one-half; then, by the maximum principle, the functions agree on the disk bounded by the circle. The identity theorem for harmonic functions now insures that these two functions agree on the full unit disk. Both of these steps, the maximum principle and the identity theorem for functions which agree on open sets, are valid for interpolation families.

THEOREM 5.1. Let $\left\{B_{z}\right\}$ and $\left\{C_{z}\right\}$ be two interpolation families with domain of definition $D$. Let $\Gamma$ be a smooth simple closed curve in $D$ such that $D_{1}$, the region bounded by $\Gamma$, is contained in $D$. If

$$
\|v\|_{B_{z}}=\|v\|_{C_{z}} \text { for all } v \text { in } \mathbf{C}^{n}
$$

holds for all $z$ in $\Gamma$, then (5.2) holds for all $z$ in $D$.

PROOF. Interpolation families are determined by their boundary values; that is, (5.2) holds on $\Gamma=\partial \bar{D}_{1}$ and hence (5.2) holds on all $D_{1}$. (This follows from the maximum principle (2.1).) Now pick $w$ in $D \backslash D_{1}$. We must show that (5.2) holds for $z=w$. Let $\Gamma_{2}$ be a simple closed curve inside $D$ so that $D_{2}$, the region bounded by $\Gamma_{2}$, contains $w$ and satisfies $D_{1} \subset D_{2} \subset D$. Select $v$ in $\mathbf{C}^{n}$. Let $F$ in $\operatorname{Hol}\left(D_{z}\right)$ be the extremal function for $v$ at $w$ with respect to the interpolation family $\left\{B_{z}\right\}$ and the domain $D_{z}$. Pick $z_{0}$ in $D_{1}$. Let $F\left(z_{0}\right)=v_{0}$. Let $\hat{v}_{0}$ be the vector $\mathbf{C}^{n}$ with $\left\|\hat{v}_{0}\right\|_{C_{z_{0}}^{*}}=1$ and

$$
\hat{v}_{0} \cdot v_{0}=\left\|v_{0}\right\|_{C_{z_{0}}} \cdot
$$

Let $G$ be the function in $\operatorname{Hol}\left(D_{z}\right)$ which is the extremal function for $\hat{v}_{0}$ at $z_{0}$ with respect to the interpolation family $\left\{C_{z}^{*}\right\}$ and the domain $D_{z}$. We now consider the scalar holomorphic function $f(z)=G(z) \cdot F(z)$. For any $z$ in $D_{1},|f(z)| \leq$ $\|G(z)\|_{C_{z}^{*}}\|F(z)\|_{C_{k}}$. On $D_{1}, B_{z}=C_{z}$ so $\|F(z)\|_{C_{z}}=\|F(z)\|_{B_{z}}$. By the extremal properties of $F$ and $G,\|F(z)\|_{B_{z}}=\|v\|_{B_{w}}$ and $\|G(z)\|_{C_{z}^{*}}=1$. Thus $|f(z)| \leq\|v\|_{B_{w}}$. At $z_{0}$

$$
\left|f\left(z_{0}\right)\right|=\left|\hat{v}_{0} \cdot v\right|=\left\|v_{0}\right\|_{C_{z_{0}}}=\left\|v_{0}\right\|_{B_{z_{0}}}=\|v\|_{B_{w}} .
$$

Thus, by the maximum principle for holomorphic functions, $f$ is constant on $D$. Hence $|G(w) \cdot v|=|G(w) \cdot F(w)|,|f(w)|=\left|f\left(z_{0}\right)\right|=\|v\|_{B_{w}} . G(w)$ is in $C_{w}^{*}$ with $\|G(w)\|_{C_{w}^{*}}=1$. Thus we have $\|v\|_{C_{w}} \geq\|v\|_{B_{w}}$. By symmetry equality must hold and the proof is complete.

Using this theorem we see that if two interpolation families have overlapping domain of definition and if they agree on the boundary of the overlap then they define a single interpolation family. More precisely, for $i=1,2$ let $D_{i}$ be simply connected domains in the plane with smooth boundary curves $\Gamma_{i}=\partial \bar{D}_{i}$. Let $D=D_{1} \cap D_{2}$ and let $\gamma=\partial \bar{D}$. $\gamma$ consists of two parts, $\gamma=\gamma_{1} \cup \gamma_{2}$, where $\gamma_{i}=\gamma \cap \Gamma_{i}, i=1,2$. 
THEOREM 5.2. For $i=1,2$ suppose $\left\{B_{z}^{i}\right\}_{z \in D_{i}}$ is an interpolation family with boundary values $\left\{B_{z}^{i}\right\}_{z \in \Gamma_{i}}$. Suppose $B_{z}^{1}=B_{z}^{2}$ for $z$ in $\gamma_{1} \cup \gamma_{2}$. Then $B_{z}^{1}=B_{z}^{2}$ in $D$ and $\left\{C_{z}\right\}$ defined by $C_{z}=B_{z}^{i}$ for $z$ in $D_{i}$ is an interpolation family on $D_{1} \cup D_{2}$ with boundary values $B_{z}^{i}$ on $\Gamma_{i} \backslash \gamma_{i}$.

The special case of this theorem in which $C_{z}$ is constant on the four sets $\gamma_{1}$, $\Gamma_{1} \backslash \gamma_{1}, \gamma_{2}, \Gamma_{2} \backslash \gamma_{2}$ yields (after a conformal map) the finite-dimensional case of the four-space reiteration theorem for complex interpolation recently proved by $\mathrm{T}$. Wolff $[\mathbf{1 0}]$. Thus we have a new perspective on his result. He obtains his result for infinite-dimensional spaces. It is not clear if Theorem 5.2 extends to that context.

VI. The reflection principle. In this section we present several versions of the reflection principle for interpolation families.

Let $D$ be the open unit disk and let $D^{+}, I, D^{-}$be the subsets of $D$ on which the imaginary part of $z$ is respectively positive, zero, and negative. We recall a version of the classical reflection principle for harmonic functions. Suppose $h$ is a real-valued function on $D^{+} \cup I$ which is continuous on $D^{+} \cup I$, harmonic on $D^{+}$ and zero on $I$. Extend $h$ by reflection to all of $D$; that is set $h(z)=-h(\bar{z})$ for $z$ in $D^{-}$. The reflection principle states that this $h$ is in fact harmonic on $D$.

Using this result for harmonic functions we can obtain results for complex interpolation families. Here is the most straightforward example. Suppose $\left\{B_{z}\right\}$ is an interpolation family on $D^{+}$which consists entirely of $l_{n}^{p}$ spaces. That is there is a function $p(z), 1 \leq p(z) \leq \infty$, on $D^{+}$such that $B_{z}=l_{n}^{p(z)}$ for $z$ in $D^{+}$.

THEOREM 6.1. Suppose $p(z)$ extends continuously to $D^{+} \cup I$ with $p(z)=2$ for all $z$ in $I$. For $z$ in $D^{-}$set $p(z)=p^{\prime}(\bar{z})\left(\right.$ where $\left.1 / p(z)+1 / p^{\prime}(z)=1\right)$. Then $\left\{B_{z}\right\}$, defined by $B_{z}=l_{n}^{p(z)}$ for all $z$ in $D$, is an interpolation family on $D$.

PROOF. $\left\{l_{n}^{p(z)}\right\}$ is an interpolation family in $D^{+}$. Thus $p(z)$ satisfies

$$
0 \leq 1 / p(z) \leq 1
$$

$1 / p(z)$ is harmonic on $D^{+}$and $1 / p(z)$ has limit $1 / 2$ on $I$. The reflection principle for harmonic functions can be applied to $1 / p(z)-1 / 2$. The extension allows us to extend $p(z)$ to all of $D$ so that $p(z)=p^{\prime}(\bar{z})$ for all $z,(6.1)$ is satisfied and $1 / p(z)$ is harmonic. Thus $\left\{B_{z}\right\}=\left\{l_{n}^{p(z)}\right\}$ is an interpolation family on all of $D$.

The only feature of the spaces $l_{n}^{p}$ used in the proof is that $\left\{l_{n}^{p(z)}\right\}$ is an interpolation family on $D$ exactly if $1 / p(z)$ is harmonic and satisfies (6.1). Such a situation is rather general. For $i=0,1$ let $A_{i}$ be an $n$-dimensional Banach space. For $\alpha, 0 \leq \alpha \leq 1$, let $A_{\alpha}=\left[A_{0}, A_{1}\right]_{\alpha}$ be the complex interpolation spaces (in the sense of A. P. Calderon) obtained by interpolating between $A_{0}$ and $A_{1}$ (see [1] for definition and details). The spaces $A_{\alpha}=l_{n}^{1 / \alpha}, 0 \leq \alpha \leq 1$, are an example. A family of Banach spaces $\left\{B_{z}\right\}$ for $z$ in a domain $E$ with all $B_{z}$ among the $A_{\alpha}$; i.e. $\left\{B_{z}\right\}_{z \in E}=\left\{A_{\alpha(z)}\right\}_{z \in E}$, will be a complex interpolation family in our sense exactly if $\alpha(z), 0 \leq \alpha(z) \leq 1$, is a harmonic function in $E$. (This is outlined in [4] and proved in [5].) Thus an analog of Theorem 6.1 holds for such a family; if $\left\{B_{z}\right\}=\left\{A_{\alpha(z)}\right\}$ is an interpolation family on $D^{+}$with $\alpha(z)$ having limiting value $1 / 2$ on $I$ then the family can be extended by reflection (i.e. so that $\alpha(z)-1 / 2=-(\alpha(\bar{z})-1 / 2))$ to be an interpolation family on all of $D$. 
Many details of the proof remain valid when the function $1 / p(z)$ (or $\alpha(z)$ ) has a limiting value other than one-half on $I$. If $\left\{B_{z}\right\}=\left\{l_{n}^{p(z)}\right\}$ is an interpolation family on $D^{+}$and $1 / p(z)$ has limit $C$ for $z$ in $I$ then $1 / p(z)$ can be extended to all of $D$ as a harmonic function by requiring $1 / p(z)-C=-(1 / p(z)-C)$ for all $z$ in $D$. If $C \neq 1 / 2$ then this extended function need not satisfy (6.1). This problem can be avoided by putting further restrictions on $\left\{B_{z}\right\}$ or by restricting attention to the subset of $D$ on which (6.1) holds. A more interesting possibility, one that is not yet fully explored, is to try to give interpolation theoretic significance to these ideas when $(6.1)$ (and more generally $0 \leq \alpha(z) \leq 1$ ) is violated. Some possibilities in this direction are indicated by Theorem 6.4 .

The previous result is a direct application of the scalar reflection principle. We now give a more general reflection principle which is more intimately connected with the interpolation theory.

For any Banach space $B$, we denote by $B^{+}$the Banach space with the norm $\left\|\left(w_{1}, \ldots, w_{n}\right)\right\|_{B^{+}}=\left\|\left(\bar{w}_{1}, \ldots, \bar{w}_{n}\right)\right\|_{B^{*}}$, where $B^{*}$ is the dual of $B$ under the bilinear pairing described in $\S 2$.

THEOREM 6.2. Suppose $\left\{B_{z}\right\}$ is a family of Banach spaces which is continuous on $\bar{D}^{+}$and an interpolation family on $D^{+}$. Suppose $B_{z}=l_{n}^{2}$ for $z$ in I. If $\left\{B_{z}\right\}$ is extended to all of $D$ by reflection by setting $B_{z}=B_{\bar{z}}^{+}$for $z$ in $\bar{D}^{-}$then the resulting family $\left\{B_{z}\right\}_{z \in \bar{D}}$ is an interpolation family on all of $D$.

Proof. Let $\left\{C_{z}\right\}$ be the interpolation family on $D$ with boundary values $C_{z}=$ $B_{z}$ for $z$ in $(\partial \bar{D}) \cap \bar{D}^{+}$and $C_{z}=B_{\bar{z}}^{+}$for $z$ in $(\partial \bar{D}) \cap \bar{D}^{-}$. For $v$ in $\mathbf{C}^{n}, v=$ $\left(v_{1}, \ldots, v_{n}\right)$, let $\bar{v}=\left(\bar{v}_{1}, \ldots, \bar{v}_{n}\right)$. We will show that for $v$ in $\mathbf{C}^{n}$ and $z$ in $D$,

$$
\|v\|_{C_{z}}=\|v\|_{C_{\bar{z}}^{+}} \text {. }
$$

Pick and fix $z_{0}$ in $D$ and $v$ in $\mathbf{C}^{n}$. Set $\mathcal{F}=\left\{F \in \operatorname{Hol}(D) ; F\left(z_{0}\right)=v\right\}, \mathcal{G}=$ $\left\{G \in \operatorname{Hol}(D) ; G\left(\bar{z}_{0}\right)=\bar{v}\right\}$. Note that the pairing of $F$ in $₹$ with $G$ in $\mathcal{G}$ given by $G(z)=\overline{F(\bar{z})}$ establishes a bijection between $\mathcal{F}$ and $\mathcal{G}$. For $F$ and $G$ related in this way,

$$
\begin{aligned}
\inf \left\{\|F(z)\|_{z} ; z \in \partial \bar{D}\right\} & =\inf \left\{\|F(\bar{z})\|_{C_{\bar{z}}} ; z \in \bar{D}\right\}=\inf \left\{\|F(\bar{z})\|_{C_{z}^{+}} ; z \in \partial \bar{D}\right\} \\
& =\inf \left\{\|\overline{F(\bar{z})}\|_{C_{z}^{*}} ; z \in \partial \bar{D}\right\}=\inf \left\{\|G(z)\|_{C_{z}^{*}} ; z \text { in } \partial \bar{D}\right\} .
\end{aligned}
$$

Thus the infimum over $\mathcal{F}$ which defines the intermediate norm for $C_{z_{0}}$ and the infimum over $\mathcal{G}$ which defines the norm at $C_{\bar{z}_{0}}^{*}$ are equal; $\|v\|_{C_{z_{0}}}=\|\bar{v}\|_{C_{\bar{z}_{0}}^{*}}$. This is equivalent to $(6.2)$.

It follows from (6.2) that for $z$ in $I$ (i.e. $z=\bar{z}$ )

$$
C_{z}=l_{n}^{2}
$$

To see this select $v$ in $\mathbf{C}^{n}$ with $\|v\|_{l_{n}^{2}}=1$. Pick $x$ in $I$.

$$
1=|v \cdot \bar{v}| \leq\|v\|_{C_{x}}\|\bar{v}\|_{C_{x}^{*}}=\|v\|_{C_{x}}=\|v\|_{C_{x}^{+}}=\|v\|_{C_{x}}^{2} .
$$

Thus $\|v\|_{l_{n}^{2}} \leq\|v\|_{C_{x}}$ for all $v$. Passing to dual norms yields $\|v\|_{C_{x}^{*}} \leq\|v\|_{l_{n}^{2}}$. When this is combined with (6.2) we obtain $\|v\|_{C_{x}} \leq\|\bar{v}\|_{l_{n}^{2}}=\|v\|_{l_{n}^{2}}$ which is the second of the two inequalities required for $(6.3)$. 
Now that $(6.3)$ is established we know that $\left\{C_{z}\right\}=\left\{B_{z}\right\}$ on $\partial \overline{D^{+}}$. Interpolation families are determined by their boundary values, thus $\left\{C_{z}\right\}=\left\{B_{z}\right\}$ for $z$ in $D^{+}$. The $\left\{C_{z}\right\}$ satisfy $(6.2)$ and by construction the $\left\{B_{z}\right\}$ satisfy the analogous condition. Thus $C_{z}=B_{z}$ for all $z$ in $D$. This shows that $\left\{B_{z}\right\}$ is an interpolation family and completes the proof.

If the family $\left\{B_{z}\right\}$ is constant on the upper semicircle $(\partial \bar{D}) \cap \bar{D}^{+}$then this result specializes to a variation of a theorem of S. G. Krein and Yu. I. Petunin on the construction of scales of Banach spaces connecting a given space $A$, a Hilbert space and the dual space of $A$ (Theorem 9.4 of $[6]$ ).

Corollary 6.3. Suppose $\left\{C_{z}\right\}$ is a continuous family of Banach spaces on $\bar{D}$ which is an interpolation family on $D$. The symmetry condition (6.2) for all $v$ in $\mathbf{C}^{n}$ and for $z$ in $\partial \bar{D}$ is the necessary and sufficient condiiion in order that $C_{z}=l_{n}^{2}$ for all $z$ in $I$.

PROOF. The previous proof shows that the condition is sufficient. On the other hand, if $C_{z}=l_{n}^{2}$ for $z$ in $I$ then we may apply the previous theorem and obtain an interpolation family $\left\{D_{z}\right\}$ which agree with $\left\{C_{z}\right\}$ on $D^{+}$and satisfies $D_{z}=C_{\bar{z}}^{+}$ on $D^{-}$. We can now apply the identity theorem, Theorem 5.1, and conclude that $D_{z}=C_{z}$ for all $z$ in $D$. These last two equalities yield (6.2) for all $v$ in $\mathbf{C}^{n}$ and all $z$ in $D$. By continuity the result extends to the boundary.

Theorem 6.2 depends on a specific choice of inner product in $\mathbf{C}^{n}$ in two ways. First, the assumption is made that for $z$ in $I, B_{z}$ equals $l_{n}^{2}$ rather than some fixed but arbitrary Hilbert space. Second, the reflection formula, $B_{z}=B_{\bar{z}}^{+}$, is tied to the particular choice of bilinear pairing used to establish the duality. These two aspects of the theorem can be changed if they are changed together. Suppose that $\left\{B_{z}\right\}$ is an interpolation family on $D^{+}$which extends continuously to $\bar{D}^{+}$and that there is some fixed Hilbert space $B$ such that $B_{z}=B$ for all $z$ in $I$. We can still apply Theorem 6.2 but we must make a preliminary "change of variables". Let $T$ be the linear map of $\mathbf{C}^{n}$ to $\mathbf{C}^{n}$ such that for all $v$ in $\mathbf{C}^{n},\|v\|_{B}=\|T v\|_{l_{n}^{2}}$. Consider the family $\left\{C_{z}\right\}$ defined on $\bar{D}^{+}$by

$$
\|v\|_{C_{z}}=\left\|T^{-1} v\right\|_{B_{z}} .
$$

$T$ is an isometry of $B_{z}$ onto $C_{z}$; symbolically $T\left(B_{z}\right)=C_{z}$. For $z$ in $I, C_{z}=$ $T\left(B_{z}\right)=T(B)=l_{n}^{2}$. Also, $\left\{C_{z}\right\}$ is an interpolation family on $D^{+}$. Thus we can apply Theorem 6.2 and extend $\left\{C_{z}\right\}$ to an interpolation family on $D$. We can then use (6.4) for $z$ in $D^{-}$to define $B_{z}$ for $z$ in $D^{-}$. The resulting $\left\{B_{z}\right\}$ will be an interpolation family on all of $D$ and will satisfy

$$
T\left(B_{z}\right)=\left(T\left(B_{\bar{z}}\right)\right)^{+} .
$$

Thus $B_{z}$ has been extended to $D^{-}$using a reflection which is the "ordinary" reflection conjugated by the change of variables which reduce the boundary spaces to $l_{n}^{2}$.

Many variations on these ideas are possible. One of the most intriguing is to use equations such as (6.4) and (6.5) with maps $T$ which are analytic but are not linear. This can yield results because many of the estimates of complex interpolation theory are based on holomorphic convexity (which is preserved by holomorphic maps) rather than geometric convexity (which would only be preserved by linear maps). 
We now give an example of a result which uses these ideas. Possible generalizations are discussed afterwards.

Theorem 6.2 certainly contains Theorem 6.1 as a special case. In the discussion after Theorem 6.1 it was noted that the scalar reflection principle could also be used to obtain results involving reflection of a family of spaces $\left\{l_{n}^{p(z)}\right\}$ across a boundary on which $p(z)$ is constant but not two. We now prove such a result using the ideas just described rather than the scalar reflection principle.

THEOREM 6.4. Suppose $\left\{B_{z}\right\}$ is a continuous family of Banach spaces on $\bar{D}^{+}$ which is an interpolation family on $D^{+}$. Suppose that for $z$ in $\bar{D}^{+}, B_{z}=l_{n}^{p(z)}$ and that $p(z)=4$ for all $z$ in $I$. Then there is an interpolation family defined on a open neighborhood of $D^{+} \cup I$ which agrees with $\left\{B_{z}\right\}$ in $D^{+} \cup I$.

ProOF. For $v$ in $\mathbf{C}^{n}, v=\left(v_{1}, \ldots, v_{n}\right)$, let $T v=\left(v_{1}^{2}, \ldots, v_{n}^{2}\right)$. For $z$ in $\bar{D}^{2}$ define $\|\cdot\|_{A_{z}}$ by

$$
\|v\|_{A_{z}}=\left\|T^{-1} v\right\|_{B_{z}}^{2} .
$$

(Although $T$ is not one-to-one, the value on the right side of (6.6) does not depend on the choice of $T^{-1} v$.) For $z$ in $\bar{D}^{+},\|v\|_{A_{z}}=\|v\|_{l_{n}^{p(z) / 2}}$ for $z$ in $S=D^{+} \cap\{p(z)>2\}$. In particular $A_{z}=l_{n}^{2}$ for $z$ in $I$. We now claim that $\left\{A_{z}\right\}$ is an interpolation family on $S$. (This follows from the realization of the $A_{z}$ as $l_{n}^{p(z) / 2}$. We wish to give a more intrinsic proof.) We will show that $\left\{A_{z}\right\}$ satisfies

$$
\text { if } F \text { is in } \operatorname{Hol}(S) \text { then } \log \|F(z)\|_{A_{z}} \text { is subharmonic on } S
$$

and

$$
\begin{aligned}
& \text { given } z_{0} \text { in } S, v \text { in } \mathbf{C}^{n} \text { there is an analytic } \mathbf{C}^{n} \text { valued } \\
& \text { function } F \text { defined near } z_{0} \text { such that } F\left(z_{0}\right)=v \text { and }\|F(z)\|_{A_{z}}
\end{aligned}
$$
is constant near $z_{0}$.

It follows from (6.7) and (6.8) that $\left\{A_{z}\right\}$ is an interpolation family on $S .\left(\left\{A_{z}\right\}\right.$ is a subinterpolation family by (6.7) and it is an easy consequence of (6.8) that the dual spaces are a subinterpolation family.)

Let $z_{0}$ in $S$ be given and let $F$ be in $\operatorname{Hol}(S)$. If $F(z)=\left(f_{1}(z), \ldots, f_{n}(z)\right)$ and $f_{1}\left(z_{0}\right) \neq 0$ for $i=1, \ldots, n$ then locally $F(z)=T(G(z))$ for an analytic $\mathbf{C}^{n}$ valued function $G(z)$. Thus, locally $\log \|F(z)\|_{A_{z}}=2 \log \|G(z)\|_{B_{z}}$ and $\log \|F(z)\|_{A_{z}}$ is subharmonic near $z_{0}$. Suppose now that some coordinate of $F(z)$ vanishes at $z_{0}$. We will consider the case $f_{1}\left(z_{0}\right)$ has a simple zero at $z_{0}$ and $f_{i}\left(z_{0}\right) \neq 0$ for $i>1$; this case will be seen to be typical. For small positive $\varepsilon, F_{\varepsilon}(z)=$ $\left(\varepsilon\left(z-z_{0}\right)^{-1} f_{1}(z), f_{2}(z), \ldots, f_{n}(z)\right)$ is analytic near $z_{0}$ and satisfies

$$
\left\|F_{\varepsilon}(z)\right\|_{A_{z}}=\|F(z)\|_{A_{z}}
$$

for $\left|z-z_{0}\right|=\varepsilon$, and for $\left|z-z_{0}\right|<\varepsilon$ satisfies

$$
\left\|F_{\varepsilon}(z)\right\|_{A_{z}} \geq\|F(z)\|_{A_{z}} .
$$

In particular (6.10) holds at $z_{0}$. The previous argument established that $\log \left\|F_{z}(z)\right\|_{A_{z}}$ is subharmonic near $z_{0}$. This combined with (6.9) and (6.10) establishes the submean value inequality for $\log \|F(z)\|_{A_{z}}$ for small circles centered at $z_{0}$. This gives $(6.7)$. 
To establish (6.8) we select $w$ in $\mathbf{C}^{n}$ so that $T w=v,\left\{B_{z}\right\}$ is an interpolation family in $D^{+}$, hence we can find $G$ in $\operatorname{Hol}\left(D^{+}\right)$with $G\left(z_{0}\right)=w$ and $\|G(z)\|_{B_{z}}$ constant. Let $F(z)=T(G(z))$. $F$ is in $\operatorname{Hol}\left(D^{+}\right), F\left(z_{0}\right)=v$, and by $(6.6),\|F(z)\|_{A_{z}}$ is constant. Thus (6.8) holds.

$\left\{A_{z}\right\}$ is an interpolation family on $S$ and equals $l_{n}^{2}$ on $I$. Thus we may use the reflection principle to extend this to an interpolation family $\left\{A_{z}\right\}$ on $S \cup I \cup\{z ; \bar{z} \in$ $S\}=R$. We now define $B_{z}$ for $z$ in $\{z ; \bar{z} \in S\}$ by $(6.6) ;\|w\|_{B_{z}}=\|T w\|_{A_{z}}^{1 / 2}$. If $A_{z}=l_{n}^{r}$ then $B_{z}$ so defined will satisfy $B_{z}=l_{n}^{2 r}$. In particular $\|w\|_{B_{z}}$ is a Banach space norm. We must now verify that $B_{z}$ is an interpolation family. If $F(z)$ is in $\operatorname{Hol}(R)$ then $\log \|F(z)\|_{B_{z}}=\log \|T(F(z))\|_{A_{z}}^{1 / 2}$. Thus $\log \|F(z)\|_{B_{z}}$ is subharmonic. This is the analog of $(6.7)$ for the $\left\{B_{z}\right\}$. We must now establish the analog of (6.8). Given $w$ in $\mathbf{C}^{n}$ and $z_{0}$ in $R$ let $v=T w$. Let $G$ be a holomorphic $\mathbf{C}^{n}$ valued function defined near $z_{0}$ so that $G\left(z_{0}\right)=v$ and $\|G(z)\|_{A_{z}}$ is constant near $z_{0}$. It is a property of such extremal functions on interpolation families of $l_{n}^{p(z)}$ spaces that their coordinate functions either vanish identically or not at all. That is $G(z)=\left(g_{1}(z), \ldots, g_{n}(z)\right)$ and each $g_{i}$ is either nonvanishing or identically zero. Thus $G(z)=T(H(z))$ for some holomorphic $\mathbf{C}^{n}$ valued function $H$. Furthermore $H(z)$ can be selected so that $H\left(z_{0}\right)=w$. This $H(z)$ is the function required to establish the analog of (6.8) and the proof is complete.

Although it is clear that the hypotheses of the theorem are unnecessarily restrictive, it is not clear what would be the full natural range of applicability of these ideas. For example, the number 4 in the hypothesis can certainly be replaced by any number $p$ with $1<p<\infty$. If, however, $p=1$ is used then the spaces $A_{z}$ will be $l_{n}^{r}$ spaces for $r<1$. In that case the theory of complex interpolation of Banach spaces as outlined in [4] is not available. However (6.7) and (6.8) would still be valid and some conclusions could be drawn. This leads to the interesting, but still relatively unexplored, area of complex interpolation of quasi-Banach spaces. Another difficulty in generalizing these ideas is related to the special considerations that were required when certain vectors (or functions) had one or more coordinates vanish. These correspond to places where the map $T$ is not locally one-to-one and hence does not have a local univalent inverse. Geometrically we were using the fact that the unit spheres of the various $l_{n}^{p}$ spaces are locally biholomorphically equivalent away from the subvariety on which the coordinate functions vanish. On this subvariety the spheres are not smooth and are not locally biholomorphically equivalent-thus different arguments are needed. The arguments which were used were particular to $l^{p}$ spaces but most of the details would go through unchanged if all the $\left\{B_{z}\right\}$ were Banach lattices.

\section{REFERENCES}

1. J. Bergh and J. Löfström, Interpolation spaces: an introduction, Springer-Verlag, Berlin, Heidelberg and New York, 1976.

2. P. L. Butzer and H. Behrens, Semi-groups of operators and approximation, Springer-Verlag. Berlin, Heidelberg and New York, 1967.

3. R. Coifman, M. Cwikel, R. Rochberg, Y. Sagher and G. Weiss, Complex interpolation for families of Banach spaces, Proc. Sympos. Pure Math., Vol. 35, Part 2, Amer. Math. Soc.. Providence. R.I., 1979, pp. 269-282. 
4. Notes in Math., vol. 779, Springer-Verlag, Berlin, Heidelberg and New York, 1980, pp. 123153.

5. _, A theory of complex interpolation for families of Banach spaces, Adv. in Math. 33 (1982), 203-229.

6. S. G. Krein and Yu. I. Petunin, Scales of Banach spaces, Russian Math. Surveys 21 (1966), 85-160.

7. A. Pelczynski, Geometry of finite dimensional Banach spaces and operator ideals, Notes in Banach Spaces (H. E. Lacy, ed.), Univ. of Texas Press, Austin, Texas, 1980.

8. R. Rochberg, Interpolation of Banach spaces and negatively curved vector bundles, Pacific J. Math. 110 (1984), 355-376.

9. R. Rochberg and G. Weiss, Derivatives of analytic families of Banach spaces, Ann. of Math. 118 (1983), 315-345.

10. T. Wolff, A note on interpolation spaces, Harmonic Analysis (Proc., Minneapolis, 1981), Lecture Notes in Math., vol. 908, Springer-Verlag, New York, 1982, pp. 199-204.

11. M. Zafran, Spectral theory and interpolation of operators, J. Funct. Anal. 36 (1980), 185-204.

Department of MAThematics, WAShington University, St. LOUis, Missouri 63130 